Radial and Nonradial Pulsations as Probes of Stellar Physics

ASP Conference Series, Vol. 259, 2002

C. Aerts, T.R. Bedding, \& J. Christensen-Dalsgaard, eds.

\title{
FCAPT Observations of Late B and Early A Supergiants and AGB S Stars
}

\author{
Saul J. Adelman ${ }^{1}$ \\ Department of Physics, The Citadel, 171 Moultrie Street, Charleston, \\ SC 29409, United States of America, e-mail: adelmans@citadel.edu
}

\begin{abstract}
I discuss single-channel differential observations of late $B$ and early A supergiants and AGB S stars obtained with the Four College Automated Photoelectric Telescope. The suggestion of periods longer than several years often seen in the seasonal mean magnitudes of both kinds of stars requires confirmation. I used Program Period98 to derive the most important frequencies for Deneb and for three AGB S stars.
\end{abstract}

\section{Introduction}

I discuss single-channel differential observations of late B and early A-type supergiants in the Strömgren uvby system and of asymptotic giant branch (AGB) $S$ stars in the Johnson $B V$ and Cousins $R I$ systems obtained with the Four College Automated Photoelectric Telescope (FCAPT) at Washington Camp, AZ, USA. The observing season is between the middle of September and the beginning of July. The photometer wheels contain 2.5 and 5.0 magnitude neutral density filters. For each group of variable, check, and comparison stars, the telescope first measures the dark count. Then in each filter it observes the sky-ch-c-v-c-vc-v-c-ch-sky where sky is a reading of the sky, ch that of the check star, $c$ that of the comparison star, and $\mathrm{v}$ that of the variable. No corrections have been made for density filter differences among the stars of each group. The comparison and check stars were chosen from supposedly non-variable stars near the variable on the sky that had similar $V$ magnitudes and $B-V$ colors. Their stability was checked using HIPPARCOS photometry (ESA, 1997).

I observed Deneb (A2 Iae) and 13 Mon (A0 Ib) for four years, HR 1035 (B9 Ia) and HR 1040 (A0 Iae) for three years, and HR 8020 (B8 Iae) for two years. They are multiperiodic with the light curves of the Ia stars having a somewhat different character each observing season. The amplitudes of the Ia stars are greater than those of $13 \mathrm{Mon}$ in agreement with Maeder (1980) and Adelman \& Albayrak (1997). Their photospheric lines in spectra of $2.4 \AA \mathrm{mm}^{-1}$ spectrograms obtained at the Dominion Astrophysical Observatory do not exhibit multiple components. But to fit the line profiles with synthetic spectral calculations, one has to use significant macroturbulence. Hence convective motions are present within their photospheres which pulsate as a unit. If the photometric

\footnotetext{
${ }^{1}$ Guest Investigator, Dominion Astrophysical Observatory, Herzberg Institute of Astrophysics, National Research Council of Canada
} 
and radial velocity variations are correlated as claimed by Paddock (1935), then we have a tool to understand the hydrodynamics of their atmospheres.

The peculiar S stars either belong to binary systems and have peculiar abundances due to mass transfer from a more evolved companion or to the asymptotic giant branch with peculiar abundances due to mixing to the surface of material from deep layers where nuclear processing has changed the original composition of the stellar material. Among the later are some of the brightest known AGB stars in our galaxy. I observed HR 3659 (RS CVn), HR 6702 (OP Her), and HR 8714 (HR Peg) for four years as well as HD 142143 (ST Her) and HR 1556 ( $\mathrm{o}^{1}$ Ori) for two years. They appear to have multiperiodic light curves.

\section{Results}

I used Period98 (Sperl, 1998) to determine the multiple periods of both types of stars. There are $337,172,185$, and 170 sets of observations of Deneb, OP Her, RS Cnc, and HR Peg, respectively. The $u v b y$ data for all five supergiants suggest the presence of periods of several years. But the different sets of bandpass values for a given star do not show the effect to the same extent. For Deneb, I derived periods in terms of decreasing amplitudes of $1513.96,587.91,12.99,67.44,13.12$, $13.71,16.78,46.21$, and 23.04 days. The longest period is not confirmable as it is the length of time over which the data were taken. But the 587.91 day period might be real. The other periods are in the range of the 16 modes of 6.9 to 100.8 days found by Lucy (1976) although none coincide with his values.

For the AGB S stars, the $B, V, R$, and $I$ light curves are almost always in phase. Their $B V R I$ data suggest the presence of periods of several years. Additional data are required for confirmation. For each star the amplitudes for $B$ and $V$ are greater than those of $R$ which are in turn greater than those of $I$. Usually the amplitude for $B$ is slightly greater than that for $V$. OP Her and HR Peg are SRB variables while RS CVn is an SRC: star with respective periods of 120.5, 50, and 120 days (Kholopov et al., 1985-8). The multiperiodic analyses of RS CVn and HR Peg both recover their known periods as the frequency with the second largest amplitude.

\section{References}

Adelman, S. J. \& Albayrak, B., 1997, IBVS, 4541

ESA, 1997, The Hipparcos and Tycho Catalogs, SP-1200

Kholopov, P.N., Samus, N.N., Frolov, M.S., et al. 1985-8, General Catalog of Variable Stars, Vol. I-III (Moscow: Nauka)

Lucy, L.B. 1976, ApJ, 206, 499

Maeder, A. 1980, A\&A, 90, 311

Paddock, G.F. 1935, Lick. Obs. Bull., 17, 49

Sperl, M. 1998, Manual for Period98: V 1.0.9, Institute for Astronomy, University of Vienna 